\title{
Die DSK stärkt die informationelle Selbstbestimmung und der EDSA gibt den Betroffenenrechten der DSGVO einen verbindlichen Rahmen
}

\section{DSK empfiehlt Nutzung der CWA zur Kontaktnachverfolgung}

Die Konferenz der unabhängigen Datenschutzaufsichtsbehörden von Bund und Ländern (DSK) hat auf ihrer ersten Zwischenkonferenz am 27. Januar 2022 eine Abkehr von der massenhaften Erfassung von Kontaktdaten empfohlen. Dazu sagte der Vorsitzende der DSK, der Bundesbeauftragte für den Datenschutz und die Informationsfreiheit (BfDI) Professor Ulrich Kelber: „Bei den aktuell sehr hohen Infektionszahlen werden die Kontaktdaten von den überlasteten Gesundheitsämtern ohnehin kaum noch genutzt. Bei niedrigen Infektionszahlen ist die allgemeine Kontaktdatenerfassung dagegen ein unverhältnismäßiger Eingriff in das Grundrecht auf informationelle Selbstbestimmung der Bürgerinnen und Bürger. Die DSK fordert deshalb, dass statt der umfassenden Sammlung von Kontaktdaten die Möglichkeiten der Corona-Warn-App (CWA) besser genutzt werden."

Die DSK setzt sich erneut dafür ein, dass in den landesrechtlichen Regelungen die CWA als datenschutzfreundliche Alternative zur Kontakterfassung vorgesehen wird. Die App warnt schnell über mögliche Risikokontakte und ist dank der dezentralen Struktur besonders sicher.

Deshalb sollten die Landesregierungen die CWA und ihre Möglichkeiten noch intensiver bewerben und so bekannter machen.

Die DSK besteht aus den unabhängigen Datenschutzbehörden des Bundes und der Länder. In diesem Jahr hat der BfDI den Vorsitz der Konferenz inne.

Die DSK hat die Aufgabe, das Grundrecht auf informationelle Selbstbestimmung zu wahren und zu schützen, eine einheitliche Anwendung des europäischen und nationalen Datenschutzrechts zu erreichen und gemeinsam für seine Fortentwicklung einzutreten.

\section{EDSA beschließt Leitlinien zum Auskunftsrecht}

Der Europäische Datenschutzausschuss (EDSA) hat am 18. Januar 2022 Leitlinien zu den Betroffenenrechten der DatenschutzGrundverordnung (DSGVO) veröffentlicht. Im Fokus steht dabei das Auskunftsrecht, mit dem Betroffene unter anderem in Erfahrung bringen können, welche Daten Unternehmen und Behörden über sie gespeichert haben.

Der Bundesbeauftragte für den Datenschutz und die Informationsfreiheit (BfDI), Professor Ulrich Kelber, begrüßt die gemeinsamen Leitlinien: „Das Recht auf Auskunft ist das grundlegende Betroffenenrecht und wird von den Bürgerinnen und Bürgern häufig in Anspruch genommen. Allerdings lässt der entsprechende Artikel der DSGVO allein einen großen Interpretationsspielraum. Der EDSA sorgt hier nun für mehr Klarheit und Einheitlichkeit."

Die Leitlinien legen insbesondere fest, welche Daten vom Auskunftsrecht erfasst sind und dass Betroffenen im Regelfall eine Kopie der Daten und nicht nur eine Zusammenfassung zu übergeben ist. Außerdem müssen die für die Datenverarbeitung Verantwortlichen angemessene Maßnahmen treffen, um Personen hinter Auskunftsersuchen zu identifizieren, damit keine unberechtigten Dritte an die Daten gelangen. Es dürfen aber auf der anderen Seite auch keine höheren Hürden aufgebaut werden, als für die Identifizierung erforderlich.

Ebenso darf ein Auskunftsersuchen beispielsweise nicht alleine unter Verweis auf einen hohen Bearbeitungsaufwand abgelehnt werden. Auch die Motivation hinter einem Auskunftsersuchen ist kein Kriterium für die Erfüllung des Auskunftsanspruchs. Die Leitlinien geben zusätzlich Hinweise und Beispiele, in welchen zeitlichen Abständen Betroffene das Auskunftsrecht gegenüber einem Unternehmen oder einer Behörde erneut geltend machen können, ohne dass ihr Ersuchen als exzessiv abgelehnt oder mit einer Gebühr belegt werden kann.

Der EDSA wird eine öffentliche Konsultation zu den Leitlinien durchführen.

Die Leitlinien finden Sie auf der Internetseite des EDSA unter https://edpb.europa.eu.

Der Bundesbeauftragte für den Datenschutz und die Informationsfreiheit (BfDI), Professor Ulrich Kelber 